\title{
EL TURISMO DESDE LA PERSPECTIVA DE LA DEMANDA. LUGAR DE ESTUDIO: PENÍNSULA DE PARAGUANÁ - VENEZUELA
}

\section{TOURISM FROM THE PERSPECTIVE OF THE DEMAND. PLACE OF STUDY: PARAGUANA PENINSULA - VENEZUELA}

\begin{abstract}
Mercy Narváez ${ }^{1}$, Gladys Fernández²
${ }^{1}$ Licenciada en Administración. Magister en Gerencia de Empresas. Doctora en Ciencias Gerenciales. Post Doctora en Ciencias Gerenciales. Universidad del Zulia. Venezuela.Email: romenarvaez@cantv.net, mercynarvaez@gmail.com² Licenciada en Contaduría Pública. Magister en Gerencia de Empresas. Doctorante en Planificación y Gestión del Desarrollo Regional. Universidad del Zulia. Venezuela. Email: hurtadoj@cantv.net
\end{abstract}

Rev. U.D.C.A Act. \& Div. Cient. 13 (2): 175-183, 2010

\section{RESUMEN}

El presente artículo, se centra en el análisis de las características de la demanda turística que selecciona a la península de Paraguaná, Estado de Falcón, Venezuela, como destino. Para tal fin, se realizó un estudio descriptivo, cuya ruta metodológica se apoyó en el empleo del enfoque cuantitativo de investigación, en el que se midieron algunos indicadores asociados a la demanda. La estrategia de recolección de datos corresponde a la empleada en los diseños de campo, por cuanto se aplicó en un contexto empírico real, mientras que el análisis de la información apeló al método de razonamiento lógico deductivo desde, el cual, se argumentaron las conceptualizaciones y demás rasgos característicos del sistema objeto de estudio. La naturaleza del diseño de investigación es, a su vez, no experimental transeccional, quedando descartado el control o el manejo deliberado de la variable a estudiar, en cualquier fase de la investigación. Los resultados del análisis permiten concluir que existen dos grupos diferenciados de turistas con características similares entre sí (un grupo orientado al turismo de recreación y otro orientado al turismo de compras y negocios). Adicionalmente, se evidencia un importante nivel de satisfacción del turista, sobre todo en lo referente a los atractivos naturales que posee el destino.

Palabras clave: Turismo, destino, segmentación de la demanda, satisfacción.

\section{SUMMARY}

This article focuses on the analysis of the characteristics of touristic demand, which selects the peninsula of Paraguaná Falcón State, Venezuela, as a destination. For this purpose, a descriptive study was performed, which methodology relied on the use of quantitative approach, from which some indicators associated with demand were measured. The data collection strategy corresponds to that used in the fieldwork design, as it was applied in a real empirical context, while analyzing the information appealed to the method of logical deductive reasoning from which conceptualizations and other characteristic features of the system under study were argued. The nature of the research design is at the same time transectional non-experimental being discarded the control or deliberate handling of the variable to consider at any stage of the research. The results of the analysis lead to the conclusion that there exist two distinct groups of tourists with similar characteristics (a recreation-oriented group and a shopping and business-oriented group). Additionally, a significant level of tourist satisfaction is evidenced, especially with regard to the natural attractions that the destination has.

Key words: Tourism, destination, demand segmentation, satisfaction. 


\section{INTRODUCCIÓN}

El turismo es definido como un sistema socio-económico basado en la recreación física y emocional de las personas, integrado por diferentes actividades que se desarrollan en la dimensión ambiental, cultural y económica del ser humano y que partiendo de la sensibilización, capacitación y organización de las comunidades para prestar buenos servicios, aprovecha racionalmente los paisajes y el patrimonio histórico cultural, para convertirlos en centros de atracción que capten visitantes nacionales o internacionales, quienes durante su estadía generan ingresos que benefician a la comunidad anfitriona (Camacaro, 2008).

Ahora bien, aun cuando el turismo tiene innumerables efectos de orden social también puede ser visto como una actividad económica por elementos definitorios, tales como la satisfacción de necesidades (ocio y recreación), los gastos y erogaciones que suponen los viajes para los turistas, el consumo y la demanda turística, la generación de riqueza, a través del proceso productivo turístico, entre otros. Es por ello que el marketing turístico debe ser capaz de comprender la demanda, es decir, conocer el tipo de turista, cómo dispondrá de su tiempo libre, cuánto tiempo dedicará a las vacaciones, cómo lo distribuirá a lo largo del año y, sobre todo, cómo efectuará la selección del destino (Pons et al. 2007).

De allí la importancia del estudio de la demanda turística de un destino, que se enfoca en conocer las características de los viajeros, relacionadas con el segmento al que pertenecen, el gasto turístico, el nivel de satisfacción de los atractivos del destino, entre otros. Vale decir, que el análisis de los rasgos distintivos de la demanda turística conllevará a diseñar acciones que propendan a mejorar la capacidad del destino para satisfacer las necesidades y deseos de ocio del turista.

En opinión de Vargas (2005), los destinos que no sean capaces de satisfacer las demandas, creando factores de diferenciación que otorgue a los turistas experiencias únicas de acuerdo con las nuevas motivaciones y comportamientos del mercado, corren el riesgo de ser excluidos del sector.

Bajo este marco de referencia, se presenta este artículo, que tiene por objeto el análisis del turismo desde la perspectiva económica de la demanda, mediante la realización de un estudio en la península de Paraguaná (espacio geográfico más septentrional de Venezuela y América del Sur), localizada en el extremo norte central del estado Falcón, de la República Bolivariana de Venezuela. Limita por el norte y al este con el Mar Caribe; al sur con el Golfete de Coro y el Municipio Miranda del estado Falcón y al oeste con el Golfo de Venezuela. Su posición geográfica se ubica en $12^{\circ} 11^{\prime} 46^{\prime \prime}$ de latitud norte y $70^{\circ} 00^{\prime} 59^{\prime \prime}$ de longitud oeste.
La península de Paraguaná, se concibe como un espacio físico territorial bien delimitado, con inmensas potencialidades, que permiten desarrollar diferentes tipos de turismo (sol y playa, ecoturismo, científico, religioso, compras y negocios), además favorecida por el Ejecutivo Nacional de Venezuela, en 1999, con la Declaratoria del Régimen Especial Aduanero de Zona Libre para la Inversión Turística y Comercial (Gaceta Oficial de la República Bolivariana de Venezuela, Decreto ํo 36.517, 14 de agosto de 1998). Dicha ley tiene como propósito promover la inversión turística en Paraguaná, a través de la aplicación de un régimen especial territorial de carácter fiscal para el fomento de la prestación de servicios en la actividad turística y comercial conexa al turismo, la cual, abarca el área geográfica comprendida por los territorios de los municipios Carirubana, Falcón y los Taques del estado Falcón (Artículo 1 de la Ley de Zona Libre).

La península de Paraguaná compite a nivel nacional con todas las regiones naturales de Venezuela, pues las mismas poseen gran cantidad de atractivos turísticos; sin embargo, dado el desarrollo de su industria turística, la isla de Margarita (Estado Nueva Esparta) es el destino por excelencia para el esparcimiento, siendo valorada nacional e internacionalmente por sus playas, su clima y su cultura, además, goza de un régimen fiscal especial que permite la comercialización de todo tipo de mercaderías importadas, exentas de impuestos. Destacan entre otros destinos turísticos, el archipiélago Los Roques, el Parque Nacional Morrocoy ubicado también en el Estado Falcón, la Selva Amazónica (Estado Bolívar), que cuenta con especiales atractivos naturales, entre ellos el Salto Ángel, los Tepuy y La Gran Sabana.

Por otra parte, entre los principales fundamentos de la teoría turística en los que se sustenta este artículo, se destacan aquellos expuestos por Mochón (2004), Gurría (2007) y Flores (2006), vinculados con los conceptos de demanda, oferta, consumo, segmentación de la demanda y motivaciones turísticas, que son reseñados a lo largo del artículo y, con los cuales, se contrastan y se complementan los resultados presentados.

\section{MATERIALES Y MÉTODOS}

Tipo de investigación: De acuerdo con la clasificación desarrollada por Hernández et al. (2003) y Méndez (2001), la investigación responde a los siguientes atributos: a) Según el área de estudio, el trabajo aparece referido al ámbito de las teorías asociadas al análisis de la variable: demanda turística. b) De acuerdo con su enfoque, la investigación propuesta es descriptiva, a partir de la cual se medirán y relacionarán el conjunto de indicadores relacionados con la variable demanda turística. 
Diseño de investigación: El presente diseño responde a las siguientes características: De campo: la estrategia de recolección de datos fue aplicada en un contexto empírico real, representado por el conjunto de visitantes-turistas de la península de Paraguaná, quienes estuvieron en esta localidad, en diciembre de 2009. No experimental: en la presente investigación no se ejerció, intencionalmente, ningún control operativo o teórico deliberado, sobre la variable a estudiar. Transeccional descriptiva: responde a que la investigación tiene como fin indagar la influencia y los valores, a través de los cuales se manifiesta el comportamiento de la variable "demanda turística", en un contexto específico y en un momento único.

Población y muestra: El universo poblacional que sirvió como unidad de análisis estuvo conformado por los turistas que visitan la península de Paraguaná; sin embargo, para efectos del estudio, se seleccionó una muestra conformada por 100 turistas, quienes visitaron la península de Paraguaná, durante la temporada vacacional de diciembre de 2009. La muestra fue consultada en relación a criterios como motivo de la visita, indicadores socio-económicos y demográficos, lugar de procedencia y nivel de satisfacción.

Técnicas e instrumentos: La técnica propuesta para la fase de campo y de la que dependió la estrategia de recolección de datos primarios fue la encuesta. La fase de campo, se apoyó, complementariamente, en diferentes técnicas, como la lectura evaluativa y la revisión de la fuente documental (literatura especializada, artículos científicos, internet, entre otras), análisis ideológico y de contenido.

En concordancia con la técnica de campo señalada, la recopilación de los datos fue a través de la aplicación de un cuestionario, elaborado para orientar el logro del objetivo de la investigación y estructurado en atención a los siguientes aspectos: motivación de la visita, variables socio-económicas y demográficas del turista, lugar de procedencia y satisfacción del visitante sobre los atractivos del destino, así como también se indagó acerca del consumo turístico.

El análisis de la información apeló al método de razonamiento lógico deductivo, desde el cual, se argumentaron las conceptualizaciones y demás rasgos característicos del objeto de estudio.

\section{RESULTADOS Y DISCUSIÓN}

Aproximación al turismo como hecho económico: El turismo es un fenómeno social, que incluye aspectos económicos, ambientales y culturales y que involucran el traslado y otras actividades que realizan las personas fuera del lugar de residencia habitual. En lo referente al aspecto económico, Ramírez (2006) señala que desde que existe pago por un servicio turístico, se generan efectos económicos e inversiones, los que originan resultados multiplicadores en la acumulación de los ingresos y que produce rentas, consumo de servicios y riqueza.

Ahora bien, para poder conceptualizar al turismo de manera general y definirlo en su alcance económico, se hace necesario establecer un marco de referencia, que incluya varios aspectos, como la demanda turística, la oferta turística, el consumo turístico, la inversión en el sector turístico, entre otros.

Las cantidades demandadas de un bien que los consumidores deseen y puedan comprar, se denomina demanda de dicho bien. En tal sentido, dentro de los bienes y los servicios que consumen los individuos cabe destacar la demanda turística. El bien denominado turismo es muy complejo, pues incluye los bienes y los servicios que consume el turista durante el periodo de tiempo que está fuera de su hogar, esto es, estancia en los hoteles, servicios de restauración, viajes, servicios de ocio, recreación y cultura, entre los principales. Los deseos que los individuos tienen de consumir turismo conforman la demanda turística (Mochón, 2004). En contraste con el autor mencionado, Parra \& Calero (2006) afirman que el enfoque de la demanda toma como punto de partida a los visitantes que consumen bienes y servicios, producidos tanto por actividades características del turismo como por otras actividades.

Cabe resaltar, que la demanda turística se produce según el área geográfica que cubran los visitantes: del exterior del país (turismo receptivo), en el interior del país (turismo interno) y hacia el exterior del país (turismo emisivo).

Por su parte, la oferta tiene que ver con los términos en los que las empresas producen y venden sus productos, y la oferta turística expresa las cantidades que los empresarios turísticos están dispuestos a ofrecer a distintos precios (Mochón, 2004).

Consecuentemente, el turismo se regula por medio del equilibrio de los dos elementos antes mencionados (la oferta y la demanda), de tal manera que si ya existe una demanda, es decir, el deseo de desplazarse a un lugar determinado, se puede crear una oferta, mediante la producción de servicios; por otro lado, si prevalece una oferta de servicios, como hoteles, playas, espectáculos, se puede crear la demanda, mediante la promoción y la venta de esos servicios (Gurría, 2007).

El fenómeno turístico, se cristaliza en el consumo de los servicios turísticos, en otras palabras, con el gasto de dinero de los viajeros o turistas en bienes y servicios antes del viaje, durante el desplazamiento propiamente dicho y después del viaje. Estos gastos, se incluyen en el concepto de consumo 
turístico. Al respecto, Mochón (2004) señala que el consumo turístico reúne una serie de características, que convierten la toma de decisión del turista en un proceso complejo, a saber:

- El turista se enfrenta a diferentes niveles de elección: duración del viaje, destino, forma de viaje, tipo de alojamiento, actividades complementarias, canal de compra.

- Requiere el desplazamiento fuera del lugar de residencia habitual de quien lo realiza.

- Supone un desembolso importante de dinero.

- La compra de un producto turístico conlleva hacer frente a la incertidumbre de adquirir algo que en parte se desconoce. El turista no sabe qué le deparará el viaje a un destino que no ha visitado antes ni la calidad de los servicios que le serán prestados.

Segmentación de la demanda turística: La demanda turística no es un todo uniforme. La diversidad de la demanda debe identificar "segmentos" de mercados que se definen como subconjuntos distintos de consumidores, que responden a características similares entre sí. Cada segmento tiene un comportamiento turístico específico en función de sus necesidades, preferencias y características. Al respecto, Ávila E Barrado (2005) expresan que hay que tener claro que el turismo no se produce para consumidores indiferenciados. El turista actual es un consumidor activo, autónomo y perfectamente diferenciado, lo que implica la necesidad de construir productos a medida para públicos, cada vez más segmentados.

Mochón (2004) plantea que los principales criterios para segmentar la demanda turística son:

- El motivo de la visita: la finalidad del viaje permite una agrupación de la demanda turística en categorías relativamente homogéneas: a) Ocio, recreo y vacaciones; b) visitas a parientes y amigos; c) negocios y motivos profesionales; d) Tratamiento sanitario; e) religión/ Peregrinación y f) otros motivos.

- Variables socio-económicas y demográficas: conocer las variables, tales como la edad, el margen de renta, el nivel de estudios, sexo, categoría profesional, estado civil, el tamaño de la familia, entre otros, es clave para programar los servicios turísticos, pues la clientela se suele segmentar en función de un grupo de estas variables.

- El lugar de procedencia: la información sobre los principales mercados emisores hacia un destino determinado contribuye a conocer el comportamiento de la demanda turística.
- Variables psicográficas: el enfoque psicológico complementa las carencias de los criterios de segmentación tradicionales.

\section{Segmentación de la demanda turística de la Península de Paraguaná}

A. Motivo de la visita. El análisis de las motivaciones puede revelar la vía, a través de la cual, las personas se plantean sus objetivos para la elección de sus destinos, reflejados tanto en la elección como en su conducta de viaje (Pons et al. 2007).

De acuerdo con Crompton, referido por Pons et al. (2007), la motivación es una acción o fenómeno que incide sobre el individuo y que produce o intenta producir una respuesta, siendo una variable muy importante en las teorías de la conducta. De esta manera, la motivación es un proceso dinámico, sinónimo de impulsar a la acción y de activar el comportamiento.

En este orden de ideas y en atención a las características con que cuenta la península de Paraguaná, se pueden desarrollar diferentes tipos de turismo, a saber (CORPOTULIPA, 2006):

Sol y playa: la península tiene $192 \mathrm{~km}$ de eje costero rodeado por el Mar Caribe y el Golfo de Venezuela, siendo un lugar privilegiado por su temperatura agradable; además, cuenta con 14 playas.

Ecoturismo: ofrece a los turistas diferentes parajes y paisajes para el disfrute ecológico.

Compras y negocios: la península goza de un régimen fiscal especial de Zona Libre para la Inversión Turística y Comercial, que le ha permitido que la ciudad de Punto Fijo se vislumbre como una urbe en pleno desarrollo económico y atractiva, tanto para los visitantes como para los inversionistas, en las áreas relacionadas a turismo y comercio, que ven en esta región un destino ideal para vacacionar y para comprar. Entre las ventajas que ofrece este régimen fiscal está la exoneración de impuestos para productos de uso personal y doméstico importados, tales como línea blanca, perfumes, ropa, electrodomésticos, calzados y licores, entre otros.

Científico: la península conjuga una serie de sitios y de paisajes naturales, con un profundo valor científico.

Religioso: Paraguaná es uno de los lugares de Venezuela con mayores edificaciones religiosas, ideales para la práctica del Turismo Religioso.

Ahora bien, en atención a la información obtenida de la población objeto de estudio, se deriva que el principal motivo por el que los turistas visitan la península es ocio, recreación 
y vacaciones, destacando el turismo de sol y de playa y el ecoturismo, dentro de los tipos de turismo preferidos por los visitantes; no obstante, otro significativo porcentaje, señala que lo motiva la adquisición de bienes (compras y negocios) (Tabla 1). Al respecto, se debe destacar que al hacer turismo existen motivaciones principales y otras accesorias o secundarias; vale decir que los viajes se realizan por un objeto (principal), pero de paso, se aprovecha para conocer la localidad y hacer otras actividades, en tal sentido, si se añaden otras motivaciones distintas de la original, una vez emprendido el viaje, se les denomina sobrevenidas.
A propósito del planteamiento anterior, Flores (2006) destaca que la diversión y el disfrute vacacional se constituyen en la más general y común motivación turística, pues toda movilización lleva consigo el deseo de divertirse, pasar un tiempo largo o corto de solaz, recreación y esparcimiento. Adicionalmente, el autor antes señalado expresa que la razón de viaje comercial, que priva sobre las personas, está firmemente adherida, en alto porcentaje, en una motivación viajera turístico-comercial, es decir, van a ejercitar su iniciativa comercial por el viaje mismo. Dicha actividad no es solo de compra-venta de productos, sino también de observación de

Tabla 1. Motivo de la visita.

\begin{tabular}{|c|c|}
\hline OPCIONES & $\%$ \\
\hline $\begin{array}{c}\text { Ocio, Recreación y Vacaciones } \\
\text { (sol y playa; ecoturismo) }\end{array}$ & 48 \\
\hline Compras y Negocios & 46 \\
\hline Actividades Científicas & - \\
\hline Actividades Religiosas & 2 \\
\hline Actividades Culturales & - \\
\hline Otros motivos & 4 \\
\hline Total & 100 \\
\hline
\end{tabular}

dichos productos (ferias industriales y comerciales), sin dejar de lado las personas que viajan por razones de compra con sentido ahorrativo.

En concordancia con lo antes descrito, el Diario El Nacional (06/12/2009) señala en un artículo titulado: "Viajeros rinden sus aguinaldos en la Zona Libre de Paraguaná”, donde las tiendas lucían abarrotadas y los vendedores y gerentes luchaban por brindar una buena atención a los clientes. Así fue el primer fin de semana en plena temporada de ventas navideñas en la Zona Libre de Paraguaná, destino de compra de muchos venezolanos, quienes buscan el ahorro, con precios libres de impuesto. En el mismo artículo, se afirma que en Paraguaná se ofrecen algunos productos a los precios más competitivos del país. Además, se señala que el visitante que se ahorra dinero en la compra, aprovecha para hacer turismo en la península, lo que trae beneficios económicos para la zona.

B. Variables socio-económicas y demográficas. El perfil medio de los turistas que se deriva de las características socio-demográficas, que se muestran en la tabla 2 , indica lo siguiente: los viajeros que frecuentan la península de Paraguaná son personas del sexo masculino (jefes de familia, en su mayoría), menores de 37 años, con estudios universitarios (profesionales), cuya estancia es significativamente corta (permanece en la península menos de dos días) y viaja acompañado por un grupo organizado (generalmente la familia), que oscila entre dos y cuatro personas. Todo ello, en concordancia con el estado civil de los visitantes, que respondieron la encuesta (principalmente casados).

Lugar de Procedencia: en relación a los datos obtenidos sobre los mercados emisores hacia el destino turístico "Península de Paraguaná", se destaca que el $100 \%$ de los visitantes son residentes del país, lo que indica que no hay presencia de un turismo receptivo, es decir, visitantes extranjeros que viajen a este nuevo destino turístico venezolano. Al respecto, señala CORPOTULIPA (2009), que la actividad turística de la península se limita al mercado nacional (los turistas proceden, principalmente, de los estados de Zulia, de Táchira y de Carabobo), con una marcada estacionalidad en las temporadas de Carnaval, Semana Santa, vacaciones escolares y Navidad. En concordancia con tal afirmación, Narváez E Fernández (2009) resaltan la inexistente afluencia turística de visitantes extranjeros a la península de Paraguaná, de lo que se infiere que la misma no es considerada como un destino turístico competitivo, a escala internacional.

Tal situación implica, necesariamente, desarrollar para la península de Paraguaná productos turísticos diferenciados 
Tabla 2. Variables socio-económicas y demográficas.

\begin{tabular}{|c|c|c|}
\hline VARIABLES & ESCALA & $\%$ \\
\hline \multirow{5}{*}{ Edad } & 18-27 años & 20 \\
\hline & 28-37 años & 36 \\
\hline & 38-47 años & 22 \\
\hline & 48-57 años & 20 \\
\hline & 58 o más & 2 \\
\hline \multirow{2}{*}{ Sexo } & Femenino & 38 \\
\hline & Masculino & 62 \\
\hline \multirow{3}{*}{ Estado Civil } & Soltero & 11 \\
\hline & Casado & 63 \\
\hline & Divorciado & 26 \\
\hline \multirow{3}{*}{ Nivel de Formación } & Media & 15 \\
\hline & Profesional & 80 \\
\hline & Postgrado & 5 \\
\hline \multirow{4}{*}{ Número de personas que le acompañan } & Viaja solo & 5 \\
\hline & 2 - 4 personas & 70 \\
\hline & 5 - 7 personas & 25 \\
\hline & 8 ó más & - \\
\hline \multirow{4}{*}{ Tiempo de permanencia } & 1 - 2 días & 55 \\
\hline & 3 - 4 días & 35 \\
\hline & 5 - 6 días & 5 \\
\hline & 7 ó más & 5 \\
\hline
\end{tabular}

y diversificados, con capacidad de satisfacer las necesidades y las expectativas de los consumidores actuales e impactar y atraer a los visitantes potenciales, tanto nacionales como extranjeros, todo ello, con el fin de obtener y luego mantener una posición competitiva en el mercado global.

Análisis descriptivo del consumo turístico de los visitantes de la Península de Paraguaná: Como complemento de los resultados presentados sobre la segmentación de la demanda turística, también se reflejan, en este artículo, datos sobre los gastos que realizan los turistas en la península, entendiendo como costes turísticos, la totalidad del dinero desembolsado por los turistas en la contratación de su viaje y durante toda su estancia. De este modo, el egreso turístico recoge los distintos gastos de desplazamiento, alojamiento, manutención, compras, excursiones, transporte público, alquiler de vehículos, ocio, entre otros, que realiza el turista. Según plantea Cabarcos Novas (2006), este desembolso constituye el indicador más adecuado para estudiar la evolución de la demanda turística. En tal sentido, el gasto promedio diario de los turistas en el destino oscila entre 600 y 800 bolívares fuertes (cuyo equivalente en divisa estadounidense, al cambio oficial en Venezuela, oscila entre 139,53 y 186,04 dólares) y la distribución de dichos gastos por categorías, se evidencian en la tabla 3, siendo los más representativos los alimentos, las bebidas y el alojamiento.

En este contexto, cabe destacar que el efecto económico es una razón importante para atraer visitantes y desplegar la actividad turística. Los gastos de los visitantes significan beneficios para los empresarios, quienes proporcionan servicios turísticos, puestos de trabajos para los residentes e ingresos para el gobierno, por concepto de impuestos $y$, en consecuencia, el desarrollo de las localidades donde se realiza dicha actividad. 
Tabla 3. Distribución del gasto turístico por categorías.

\begin{tabular}{|c|c|}
\hline CATEGORÍA DE GASTO & $\begin{array}{c}\text { PROPORCIÓN } \\
\text { PROMEDIO (\%) }\end{array}$ \\
\hline Alojamiento & 36 \\
\hline Alimentos y Bebidas & 40 \\
\hline Recreo y Diversiones & 10 \\
\hline Transporte & 12 \\
\hline Otros gastos & 2 \\
\hline
\end{tabular}

Grado de satisfacción del turista sobre los diversos atractivos de la península de Paraguaná, como destino turístico: Todo lugar que constituya un destino turístico debe tener una serie de características que motiven el desplazamiento temporal de los viajeros. Estas características, desde el punto de vista del visitante, se denominan atractivos turísticos, pues son los que llaman y atraen su atención (Gurría, 2007). Para que un espacio geográfico sea denominado "destino turístico" tiene que ofrecer un conjunto de particulares que atraigan al turista por todo lo que pueda obtener de él y por la satisfacción de las necesidades del mismo, sean temporales o permanentes.

De la lectura de la tabla 4, se deriva que los atractivos que más satisfacción causan en los informantes, objeto de esta investigación, son: la amabilidad de los residentes, las playas, el clima, el ambiente agradable y el paisaje. Además, cabe destacar que los precios de los artículos que se comercializan en la península se constituyen en un verdadero atractivo, pues tal como lo señala el artículo: "Paraguaná es una marca turística" (El Nacional, 07/03/2010), la compra de productos en la Zona Libre de Paraguaná representa un importante elemento de atracción para los viajeros; sin embargo, en el mismo artículo, se señala la necesidad de diversificar la inversión, con el propósito de brindarles a los visitantes más posibilidades de esparcimiento y de recreación, para complementar la oferta turística. No obstante, se debe destacar que también existen algunos aspectos que se constituyen como no satisfactorios para los visitantes del destino, tales como: los servicios públicos y la seguridad ciudadana.

Tabla 4. Satisfacción del turista sobre los diversos atractivos.

\begin{tabular}{|c|c|c|}
\hline ATRACTIVOS TURISTICOS & SATISFACTORIO & INSATISFACTORIO \\
\hline Playas & 90 & 10 \\
\hline Clima & 90 & 10 \\
\hline Vida Nocturna & 60 & 40 \\
\hline Paisajes & 85 & 15 \\
\hline Precios de los artículos comercializados en la Zona Libre & 95 & 5 \\
\hline Servicios de Restauración (Alimentos y Bebidas) & 45 & 55 \\
\hline Gastronomía & 75 & 35 \\
\hline Servicios de Hospedaje & 60 & 40 \\
\hline Gente amable & 95 & 5 \\
\hline Seguridad Ciudadana & 30 & 70 \\
\hline Servicios Públicos & 25 & 75 \\
\hline Ambiente Agradable & 90 & 10 \\
\hline
\end{tabular}


De forma detallada, las conclusiones que se extraen del presente estudio, se pueden puntualizar de la siguiente manera:

- El perfil del turista que visita la península de Paraguaná corresponde con un visitante nacional con una marcada tendencia al uso intensivo del destino en las temporadas de diciembre, Carnaval, Semana Santa y agosto, cuya estancia es de menos de dos días y viaja acompañado por su grupo familiar, el cual, oscila entre dos y cuatro personas.

- Los resultados del análisis de segmentación indican la existencia de dos grupos diferenciados de turistas, con características similares. Un grupo orientado al turismo de ocio, de recreación y de vacaciones (sol y playa, ecoturismo) y, otro, orientado al turismo de compras y negocios, que busca aprovechar las ventajas del régimen fiscal especial de Zona Libre para la Inversión Turística y Comercial, del cual goza la península.

- Se evidencia un importante nivel de satisfacción del turista - visitante de la península, sobre todo, en lo referente a los atractivos naturales que posee el destino; sin embargo, se estima necesario la creación y la adecuación de una oferta turística que incluya atractivos culturales, religiosos, científicos y comerciales, para de esta manera atraer, atender y diversificar la demanda que, progresivamente, se ha venido generando. De igual forma, las organizaciones de carácter público deben sumar esfuerzos para solventar la debilidad que existe en materia de infraestructura (vialidad, transporte público, edificaciones) y servicios básicos (agua, electricidad, aseo), con el fin de convertir a Paraguaná en un destino turístico competitivo, que sea capaz de satisfacer las necesidades de los residentes y los visitantes, además, incrementar las posibilidades de selección de la Península de Paraguaná, como destino turístico, por parte de los viajeros nacionales y extranjeros.

- Adicionalmente, y en correspondencia con los resultados obtenidos en este estudio, se estima conveniente la formulación e implementación de directrices públicas de ordenación, de planificación, de promoción y de regulación de la actividad turística que se desarrolla en la península, destacando que no sólo es tarea de los gobiernos (locales, regionales y nacionales) implantar dichas directrices, sino que se deben involucrar todos los actores vinculados al turismo, tales como el sector privado, las organizaciones no gubernamentales y la comunidad en general, con el propósito de crear disposición política y consenso social, que permita la ejecución de acciones que resulten realmente efectivas para la gestión eficiente, equilibrada y sostenida del destino.
Conflictos de intereses: El manuscrito fue preparado y revisado con la participación de todos los autores, quienes declaramos que no existe ningún conflicto de intereses que ponga en riesgo la validez de los resultados presentados. Financiación: Este estudio fue financiado por el Consejo de Desarrollo Científico y Humanístico (CONDES), de la Universidad del Zulia, Venezuela.

\section{BIBLIOGRAFÍA}

1. AVILA, R; BARRADO, D. 2005. Nuevas tendencias en el desarrollo de destinos turísticos: Marcos conceptuales y operativos para su planificación y gestión. Cuadernos de Turismo. (España). Número 015:27-43.

2. CABARCOS NOVAS, N. 2006. Promoción y venta de servicios turísticos. Comercialización de servicios turísticos. Ideaspropias Ed. S.L. España. 304p.

3. CAMACARO, A. 2008. Turismo Básico: Un enfoque integral. Editorial Biosfera C.A. Venezuela. 255p.

4. CORPORACIÓN PARA LA ZONA LIBRE PARA EL FOMENTO DE LA INVERSIÓN TURÍSTICA EN LA PENÍNSULA DE PARAGUANÁ (CORPOTULIPA). 2006. Disponible desde Internet en: http://www.corpotulipa.gob.ve (con acceso el 11/01/10).

5. CORPORACIÓN PARA LA ZONA LIBRE PARA EL FOMENTO DE LA INVERSIÓN TURÍSTICA EN LA PENÍNSULA DE PARAGUANÁ (CORPOTULIPA) 2009. Zona Libre de Paraguaná como instrumento del Estado para alcanzar un modelo productivo de beneficio social. CORPOTULIPA. 48p.

6. EL NACIONAL. Domingo 6 de diciembre de 2009. "Viajeros rinden sus aguinaldos en la Zona Libre de Paraguaná". Regiones. Ciudadanos. p.7.

7. EL NACIONAL. Domingo 7 de marzo de 2010. "Paraguaná es una marca turística”. Regiones. Ciudadanos. p.5.

8. FLORES, M. 2006. Introducción a la teoría y técnica del turismo. Vadell Hermanos Editores C.A. Caracas. 195p.

9. GURRÍA, M. 2007. Introducción al Turismo. Editorial Trillas. México.136p.

10. HERNÁNDEZ, R.; FERNÁNDEZ, C.; BAPTISTA, P. 2003. Metodología de la Investigación. Editorial Mc. Graw Hill. México. 501p. 
11. MÉNDEZ, C. 2001. Metodología. Diseño y Desarrollo del Proceso de Investigación. III Edición. McGrawHill Interamericana de España, S.A. 246p.

12. MOCHÓN, F. 2004. Economía y Turismo. McGraw-Hill/ Interamericana de España, S.A.U. Madrid. 423p.

13. NARVAEZ, M; FERNANDEZ, G. 2009. Indicadores de competitividad para destinos turísticos en el marco de la sostenibilidad: Un análisis aplicado a la península de Paraguaná. Revista Venezolana de Análisis de Coyuntura. 15(2):101-119.

14. PONS, R.; MORALES, L.; DÍAZ, Y. 2007. La imagen del destino y el comportamiento de compra del turista. Revista Teoría y Praxis. (México). 3(4):89-102.

15. PARRA, E.; CALERO, F. 2006. Gestión y Dirección de Empresas Turísticas. España: Mc Graw Hill. 516p.
16. RAMÍREZ, C. 2006. Visión integral del turismo. Fenómeno dinámico universal. Editorial Trillas, S.A. de C.V. México. 239p.

17. REPÚBLICA BOLIVARIANA DE VENEZUELA. 1998. Ley de Zona Libre para el fomento de la Inversión Turística y Comercial en la Península de Paraguaná. Gaceta Oficial No. 36.517, Agosto 14,1998.

18. VARGAS, E.E. 2005. Innovación y competitividad: Reto para las pequeñas empresas turísticas. En: Frausto, $\mathrm{O}$. (Ed.). Desarrollo sustentable: Turismo, costas y educación. Cozumel: Universidad de Quintana Roo. Disponible desde Internet en: http://www.teoriaypraxis.uqroo. $\mathrm{mx} /$ doctos/publicaciones/Desarrollo-sustentable.pdf (con acceso el 11/01/10).

Recibido: Abril 26 de 2010

Aceptado: Septiembre 29 de 2010 